\title{
Possible Relief for Migraine Patients: Saussurea Lappa
}

\author{
Wadiah S. Backer ${ }^{1}$, Amin M. Aljefri ${ }^{2}$, Nagwa Al Sawi ${ }^{1}$ and Lina Albaz ${ }^{1}$ \\ 1. Biochemistry Department, King Abdulaziz University, Saudi Arabia \\ 2. King Abdulaziz Hospital, Saudi Arabia
}

\begin{abstract}
This study conducted a clinical trial to evaluate the relieving effects of Saussurea lappa on migraine patients. The results showed that calcium and magnesium concentrations decreased significantly in patients while serotonin increased after two weeks of treatment with Saussurea lappa. The drug (Amitriptyline 20mg/day and Ibuprofen 400mg/day) decreased alkaline phosphatase level significantly, while Saussurea lappa had no effect on its level. This study found Saussurea lappa a safe and effective alternative therapeutic agent which indicated that this root could have an effect in relieving headaches (migraine).
\end{abstract}

Key words: Migraine, saussurea lappa, serotonin, calcium and magnesium.

\section{Introduction}

Migraines, which affect about $10-15 \%$ of the population in various countries, has a long history with insufficient understanding and uncertain etiology. The condition may occur at any age, but its prevalence increases from childhood up to 40 years of age. Migraines are more common in women than in men. According to an American study, the migraine gender ratio (M:F) is 1:2.9 [1], while another study in Saudi Arabia found a gender ratio of (M:F) of 1:3.9 [2].

Migraine remains one of the most undertreated neurological conditions. As a result, many migraineurs express dissatisfaction with treatment and discontinue it, despite continued unbearable migraines. The side effects considered serious in $1.7 \%$ of those treated. The treatment of migraine has not only medical but also serious economic and social implications. The economic burden of migraine headaches is tremendous, as measured through individual loss of productivity [1-2].

Migraine is defined as lateralized or generalized pain in the head, which can feel dull or trooping. It is building up gradually and may last for several hours or

Corresponding author: Wadiah Saleh Backer, assocaite professor, research fields: enzyme and natural products. E-mail: wbacker@kau.edu.sa. longer. Migraine is often accompanied with anorexia, nausea, vomiting, photophobia, phonophobia, and blurring of vision [3].

The plant Saussurea lappa, known as costus, is found extensively in the northwest and northeast sub-Himalayan regions along rivers. Their roots are used for medicinal purposes [4]. Costus has two varieties: Indian costus, black, thick, hot and bitter; and sea costus, white, light and pleasant smelling. In spite of their differences, both carry the same therapeutic value [5].

The pathogenesis of migraine focuses on serotonin theory [6]. Serotonin, a neurotransmitter, appears to be a critical factor in the processes leading to migraines. Serotonin (also called 5-hydroxytryptamine or 5-HT) is involved in regulation of pain perception and depression, among other important functions. A number of studies have suggested that serotonin serves as a brake in the migraine process. A decline in serotonergic function has been noted during acute migraine attacks. Serotonin appears to block the peptides involved in over stimulating nerves and producing inflammation [6-7]. Platelets comprise an important etiological consideration in migraines, as they contain over $90 \%$ of the serotonin in the blood. Once they aggregate, platelets release serotonin, causing a potent vasoconstrictive effect [8] (Fig. 1). 


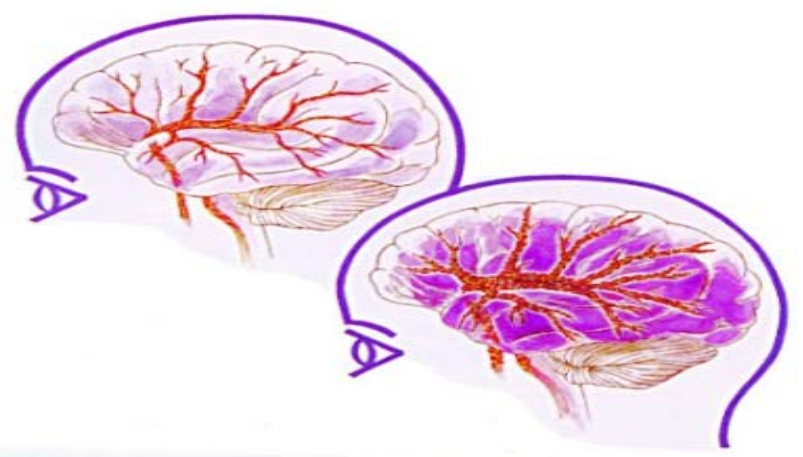

Fig. 1 Inflamed blood vessels causing migraine: The head at the top shows the brain before a migraine. The one at the bottom shows how the blood vessels become inflamed, irritating the nerve endings around them and causing pain (Simon, 2003).

A large number of clinical and experimental studies have clearly established the importance of magnesium in the pathogenesis of migraine headaches. However, the precise role of various effects of low magnesium levels in the development of migraines remains to be discovered. Magnesium concentration has an effect on serotonin receptors, neurotransmitters, and other migraine related receptors. The available evidence suggests that up to $50 \%$ of patients suffering acute migraine attacks have lowered levels of ionized magnesium. Injection of magnesium results in a rapid and sustained relief of an acute migraine in such patients [9].

\section{Objective}

From ancient times, physicians and others have used Saussurea lappa (costus) to relieve headaches as mentioned in the literature of Ibn-al-Qayyim and Al-Imam Al-Thahabi. However, medical professionals have not used it extensively due to lack of scientific research required by contemporary science. On the other hand, many recent studies have tried to understand its pathogenesis. Despite of that, there still have no cure for migraine and no satisfactory treatment. Therefore, this study attempts to find the possible effects of Saussurea lappa on migraines as an effective, safe, and inexpensive treatment.

\section{Methods}

The study was performed with Saussurea lappa roots harvested from India and purchased from a market in Jeddah, Saudi Arabia.

Before treatment with Saussurea lappa patients responded to a simple case-study questionnaire to ensure that they satisfied the inclusion criteria and to determine the severity of their symptoms. After two weeks of treatment, patients answered the same questionnaire about the degree of their migraine symptoms.

The 32 drug- and Saussurea lappa-treated patients (29 females and 3 males ranging in age from 19 to 48) answered the case-study questionnaires. The first question (Has a headache limited your activities for a day or more?) was answered "Yes" by $94 \%$ of the patients and "No" by 6\%. The second question (Are you nauseated or sick to your stomach when you have the attacks?) was answered "Yes" by $70 \%$ of the patients and "No" by 30\%. The last question (Does light bother you when you have a headache?) was answered "Yes" by 78\% of the patients and "No" by $22 \%$. Chi-Square test was performed to demonstrate the relationship between the severity of the migraine attacks and the degree of improvement after treatment with Saussurea lappa (1 g/500 ml/day) for two weeks.

The clinical trial was performed in accordance with the recommendations guiding biomedical research involving human subjects adopted by the Bioethical and Research Committee of King Abdulaziz University Hospital. The research has been reviewed and approved by the committee with informed consent given to the patients prior to inclusion in the trial.

After collecting blood samples (before and after two weeks of treatment) from the migraine patients and healthy individuals, at the King Abdulaziz University Hospital Labs, the assessment of the following has been done: liver function (total bilirubin TBIL, alanine aminotransferase ALT, $\gamma$-glutamyltranserase GGT, aspartate aminotransferase AST and alkaline phosphatase ALP); kidney function (total protein, 
albumin, uric acid); hematology parameters (white blood cell count WBC, red blood cell count RBC, hemoglobin $\mathrm{Hb}$, hematocrit HCT, mean cell volume MCV, mean corpuscular hemoglobin $\mathrm{MCH}$, mean corpuscular hemoglobin concentration MCHC, red blood cell distribution width RDW and platelet PLT); calcium; phosphorus; and magnesium. Serotonin in the plasma was determined by the basic principle of competitive ELISA [10].

\section{Results}

The clinical trial results of the liver and kidney function parameters, hematology parameters, calcium, phosphorus, magnesium, and serotonin for the three groups: control, migraine patients treated with Saussurea lappa (1 g/500 ml/day), and treated with drugs (Amitriptyline $20 \mathrm{mg} /$ day and Ibuprofen 400 $\mathrm{mg} /$ day) for two weeks are as follows.

On the liver function parameters: total bilirubin, alanine aminotransferase, $\quad \gamma$-glutamyltranserase, aspartate aminotransferase had no apparent significance, except for alkaline phosphatase (ALP). Results showed that the ALP concentration in controls not treated with Saussurea lappa was significantly $(\mathrm{P}<$ 0.05 ) higher by $40 \%$ and $27 \%$ than both drug and Saussurea lappa-treated groups, respectively. Meaningfully, migraine patients had lower concentrations of ALP than healthy individuals. In addition, migraine patients treated with Saussurea lappa had a significant $(\mathrm{P}<0.05)$ high concentration of ALP (30\%), compared with the drug-treated group (Fig. 2).

On kidney function and hematology parameters showed no significant changes in concentration before or after treatment with Saussurea lappa.

High calcium concentrations are generally associated with headaches, including migraines. The results indicated a significant $(\mathrm{P}<0.05)$ decrease in calcium concentration by $20 \%$ in migraine patients after treatment with Saussurea lappa. On the contrary, calcium concentration in the control group increased by $17 \%$ after treatment with Saussurea lappa. Also, results showed that controls had high calcium concentration, $15 \%$, compared with patients after treatment with Saussurea lappa (Fig. 3). This could indicate that Saussurea lappa helps migraine patients by lowering their calcium concentrations, and this could relieve migraine symptoms.

The concentration of phosphorus in controls significantly $(\mathrm{P}<0.05)$ high by $18 \%$ after treatment with Saussurea lappa. The phosphorus concentration in migraine patients also increased after treatment with Saussurea lappa. The result reveals that the control group generally had a high concentration of phosphorus compared with all groups (migraine patients treated with Saussurea lappa and migraine patients treated with drugs).

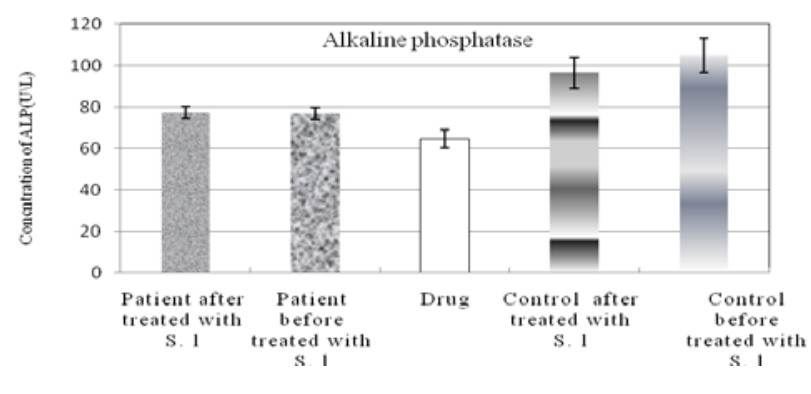

Fig. 2 Effect of treatment with Saussurea lappa on the levels of alkaline phosphatase (ALP): Control and migraine patient treated with Saussurea lappa (S.l) $(1 \mathrm{~g} / 500 \mathrm{ml})$ and migraine patient treated drugs (Amitriptyline $20 \mathrm{mg} /$ day and Ibuprofen $400 \mathrm{mg} / \mathrm{day}$ ) for two weeks. Data are mean of the concentration of ALP (from 22 migraine patients, 10 controls and 10 drug treated patients; each sample was assayed in duplicate \pm SE).

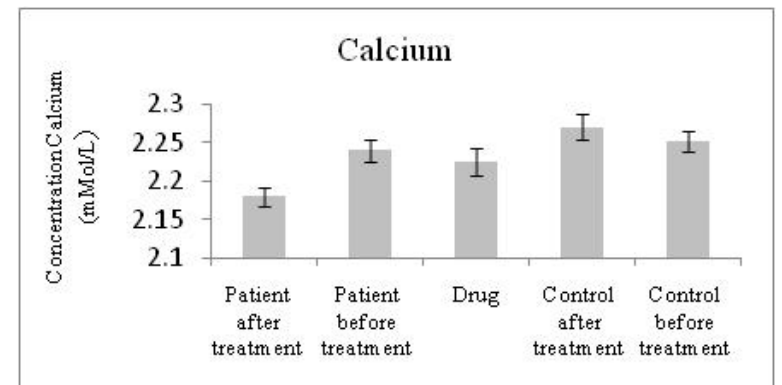

Fig. 3 Effect of treatment with Saussurea lappa on the levels of calcium: Control and migraine patient treated with Saussurea lappa (S.I) (1 g/500 ml) and migraine patient treated drugs (Amitriptyline $20 \mathrm{mg} /$ day and Ibuprofen 400 $\mathrm{mg} /$ day). All groups treated for two weeks. Data are mean from 22 migraine patients and 10 controls and 10 drug treated patients; each sample was assayed in duplicate \pm SE. 
Magnesium concentration decreased in migraine patients and increased significantly $(\mathrm{P}<0.05)$ in the control group after two weeks of treatment with Saussurea lappa (Fig. 4).

Serotonin results revealed that serotonin concentration decreased in migraine patients after treatment with Saussurea lappa for two weeks (Fig. 5). On the other hand, concentration of serotonin increased in the control group after treatment with Saussurea lappa. Both decreasing and increasing serotonin concentration after treatment had no statistical significance. This could be due to the fact that the period of the treatment was not long enough to show real significance, or the patients size were not large enough.

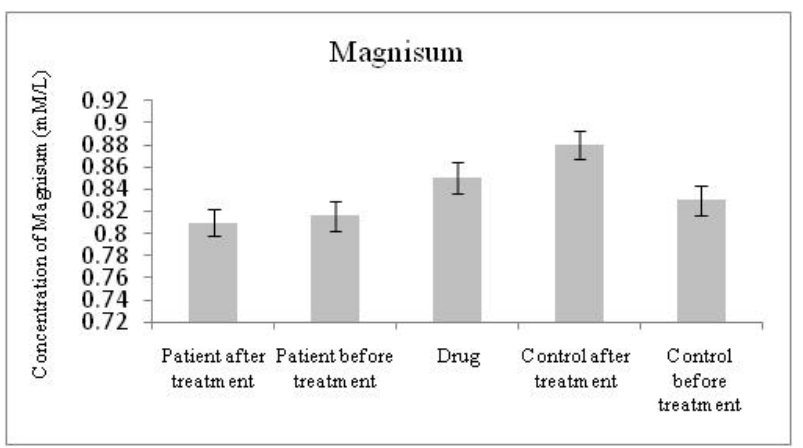

Fig. 4 Effect of treatment with Saussurea lappa on the levels of magnesium. Control and migraine patient treated with Saussurea lappa (S.I) (1 g/500 ml) and migraine patient treated drugs (Amitriptyline $20 \mathrm{mg} / \mathrm{day}$ and Ibuprofen 400 mg/day.

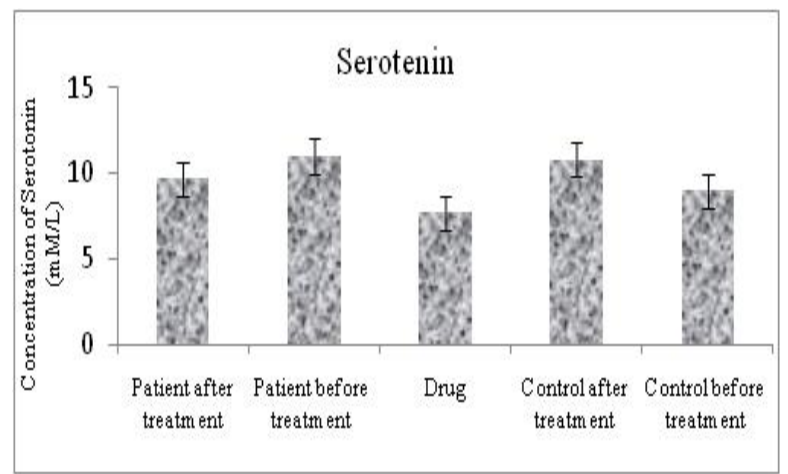

Fig. 5 Effect of treatment with Saussurea lappa on the levels of serotonin: Control and migraine patient treated with Saussurea lappa (S.l) $(1 \mathrm{~g} / 500 \mathrm{ml})$ and migraine patient treated drugs (Amitriptyline $20 \mathrm{mg} / \mathrm{day}$ and Ibuprofen 400 $\mathrm{mg} /$ day). All groups were treated for two weeks. Data are mean from 22 migraine patients and 10 controls and 10 drug treated patients; each sample was assayed in duplicate \pm SE.
The results of the relationship between the severity of the migraine attacks and the degree of improvement after treatment with Saussurea lappa (1 g/500 ml/day) for two weeks, showed clearly that the patients improved after two weeks of treatment with Saussurea lappa, but the value of the Chi-Square test was 1.4 with p value 0.2 which considered insignificant (Fig. 6).

\section{Discussions}

The result of alkaline phosphatase (ALP) agrees with Chernecky and Berger [11] and Pagana and Pagana [12], which found that prolonged use of alleviated drugs may cause low levels of ALP in individuals, indicating low-functioning adrenal glands, protein deficiency, malnutrition or more commonly, zinc deficiency. While the levels of ALP showed no significant change in migraine patients before and after treated with Saussurea lappa (Fig. 2). This confirms that Saussurea lappa may be safe for liver function.

Research has found that $50 \%$ of all migraine sufferers have low magnesium and high calcium levels. Calcium-channel blockers, commonly used to treat heart disease, are important drugs for preventing headaches [13] by lowering the concentration of calcium as Saussurea lappa revealed in this study.

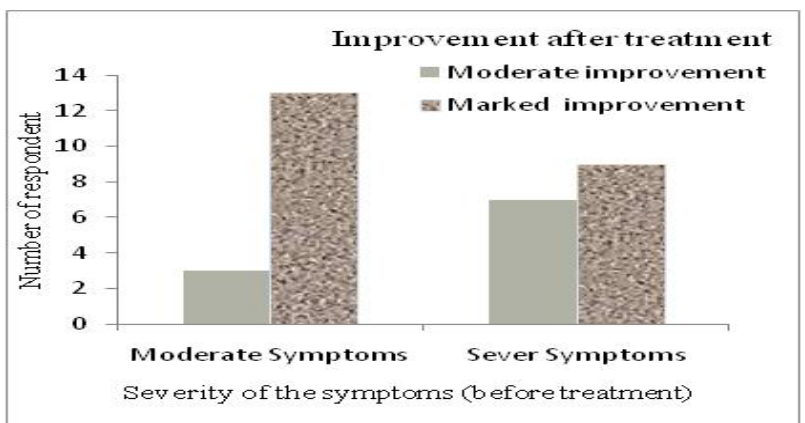

Fig. 6 Chi-Square test: For demonstrating the severity of the symptoms before and after treatment. It showed the relation between the severity of the attacks and the range of improvement after treatment with Saussurea lappa (1 $\mathrm{g} / 500 \mathrm{ml} /$ day). Patients of moderate and sever symptoms showed after treatment with Saussurea lappa moderate or marked improvement. Data are mean of 32 migraine patients. 
According to the result of magnesium, it may be used as an alternative supplement for migraine sufferers who cannot tolerate medications due to side effects or who cannot take migraine medications due to pregnancy or heart disease. Our results support previous findings of Mauskop and Altura [14]. Magnesium concentration has an effect on serotonin receptors and other migraine-related receptors. The available evidence suggests that up to $50 \%$ of patients have low levels of ionized magnesium during an acute migraine attack. When compared with normal subjects, migraine patients had low levels of serum magnesium. Serum magnesium levels tended to be further reduced during attacks, which could represent an expression of reduced cerebral magnesium levels and which would contribute to defining the threshold for migraine attacks. Low red-blood-cell magnesium levels could indicate reduction of brain magnesium concentration observed in migraine patients [9, 15].

The value of the Chi-Square test was 1.4 with $\mathrm{p}$ value 0.2 which considered insignificant. This could be due to the fact that the treatment did not last long enough to show real significance, or the sample size was not large enough to represent the population.

Saussurea lappa root contains several minerals and phenolic compounds and thus may be used as an antioxidant and safe therapeutic agent for many diseases and disorders including migraines [16].

\section{Conclusion}

Saussurea lappa helps migraine patients by: decreasing their calcium, magnesium, serotonin and increasing phosphorus, and alkaline phosphatase concentrations. This could relieve migraine symptoms. Also, the results may indicate that Saussurea lappa could be considered a safe herb for liver, kidneys, immune system and bone marrow in all subjects.

\section{References}

[1] S. Dzugan, EL Magazine 2004.

[2] A. C. Longe, Jornal of King Saud University, Abha Branch 1998.

[3] P. Kumar and M. Clarck, Clinical Medicine, 2002, p. 1202.

[4] L. A. Khan, Islam Voice 2001, (14), 159.

[5] I. Q. Al-Jawzeyah, Al-Teb Al-nabawy, Dar Al-Hadith, Cairo, 2001, p. 241.

[6] Z. Bajwa and A. Sabahat, Uptodate 2005, (13).

[7] H. Simon, Harvard Medical School Publications 2003.

[8] K. Kovac, L. Tothfaulsi, F. Herman and K. Magyar, Pol. J. Pharmacol.Pharm. 1998, (40), 691-695.

[9] K. Mishima, Takeshima T., Shimomura T., Kitano A., Takahashi K., Nakashima K. and O. H, Life Extention 1997, (37), 4-561

[10] D. K. Lahiri, Y. W. Ge, E. H. Sherman and S. C. Bondy, Age-related changes in serum melatonin in mice: Higher levels of combined melatonin and 6-hydroxymelatonin sulfate in the cerebral cortex than serum, heart, liver and kidney tissues, J. Pineal Ret 36 (2004) 217-223.

[11] C. C. Chernecky and B. J. Berger, Laboratory Tests and Diagnostic Procedures, Saunders, Philadelphia, 2004, pp. 52-56.

[12] Pagana and Pagana, Mosby’s Manual of Diagnostic and Laboratory Tests, Mosby, St. Louis, 2006.

[13] A. G. Sharpe and E. Catherine, Inorganic Chemistry, Prentice Hall, 2004, p. 949.

[14] A. Mauskop and B. Altura, Clin Neurosci 5 (1998) 24-27.

[15] N. Ramadan and T. Buchanan, Clinical Neurophysiology 118 (2006) 2297-2304.

[16] M. Pandey, S. Rastogi, A. Kumar and S. Rawat, Journal of Ethnopharmacology 110 (2007) 379-390. 